\title{
Lovastatin Nanoparticle Synthesis and Characterization for Better Drug Delivery
}

\author{
A. Seenivasan ${ }^{1}$, Tapobrata Panda*, ${ }^{*}$ and Thomas Théodore ${ }^{2}$ \\ ${ }^{1}$ Biochemical Engineering Laboratory (MSB 140A and MSB 235A), Department of Chemical Engineering; ${ }^{2}$ Department \\ of Mechanical Engineering, Indian Institute of Technology Madras, Chennai-600 036, Tamil Nadu, India
}

\begin{abstract}
Nano drug delivery system is the latest technology employed in various medicinal applications. This technology can be adapted to the conventional drug administration due to its site-specific targeting phenomena. The oral lipophilic drug administration has its drawbacks due to poor solubility and bioavailability. Lipid-based carrier systems are now widely popular due to improved efficiency, especially for lovastatin delivery. Lovastatin is an important drug which arrests the rate-limiting step of the cholesterol cascade. This drug has short half-lives, poor oral-administered bioavailability, poor solubility, and is rapidly metabolizable. Based on the composition, the drug delivery carriers are classified into solid lipid nanoparticles (SLNs), lipid emulsions (LEs), and nanostructured lipid carriers (NLCs). Among them, NLCs are a smarter generation of drug delivery carriers for lovastatin. The selection of various lipid systems and their formulation are discussed in this paper. Moreover, the characterization of these carrier systems to achieve the optimal characteristic features is discussed in a concise manner.
\end{abstract}

Keywords: Lovastatin, nanoparticles, solid lipid nanoparticles, lipid emulsions, nano-structured lipid carriers.

\section{INTRODUCTION}

The drugs conceptualized from combinatorial chemistry research and/or the drugs selected from biologically-based screening techniques are quite often lipophilic in nature. The potential problems associated with conventional oraladministrated system (tablets) are non-specific targeting, side effects due to high dosage, and poor stability. Moreover, there are many barriers for the oral-administered drug to reach proper target sites especially for poorly soluble drugs. The development of a suitable carrier system for the optimal oral administration of these drugs is a challenging task till date due to the complex nature and high variability of the host and drug system. Carrier development for the parenteral administration of these drugs has many problems including the massive use of surface-active excipients for solubilization [1]. Nano-aided drug delivery systems are a promising avenue in the field of targeted drug delivery for the treatment of various diseases. The application of this technology improves the bioavailability, physico-chemical stability, and drug loading efficiency. Surface coating, nanoparticle fabrication, fundamental composition, and drug loading are critical factors that need to be considered during drug design [2, $3]$. Examples of this delivery system were successfully employed in the field of brain, cosmetic, dermal, bone fracture, and cancer target delivery [2-5].

Nano-aided drug delivery systems can generally be divided into two groups, viz., polymeric and lipidic systems. Polymer-aided nanoparticles in the market are limited by

*Address correspondence to this author at the Biochemical Engineering Laboratory (MSB 140A and MSB 235A), Department of Chemical Engineering, Indian Institute of Technology Madras, Chennai 600 036, Tamil Nadu, India; Tel: +91-9840708563; E-mail: panda@iitm.ac.in some of the characteristic features, viz., toxicity of the polymers, presence of solvent residues in the compound during production and purification, high cost, degradability, lack of suitable large-scale production units, requirement of high quality and purity in the case of biodegradable polymer [6, 7]. Lipid-based carriers such as solid lipid nanoparticles (SLNs), nano-structured lipid carriers (NLCs), and lipid emulsions (LEs) are chosen for the recent application because of the drawbacks of the polymeric support. These systems demand suitable conditions for drug delivery, viz., the use of physiologically tolerated lipids, large-scale production, protection of drugs from degradation, improved bioavailability, minimum level of toxicity, and controlledrelease characteristics. Lipid carrier systems have been used in vivo in the medicinal application of DNA/RNA due to its low cytotoxicity [3].

Lovastatin, a cholesterol-lowering agent, is isolated from strains such as Aspergillus, Monascus, and Streptomycetes species. This compound is highly effective for patients with moderate hypercholesterolemia [8]. The lactone form of this drug is inactive (proactive) and is converted by cytochrome P450 3A4 to the pharmacologically active free hydroxy acid form. This drug is analogous to HMG-CoA, which competitively binds with HMG-CoA reductase. In this process, the drug makes the enzyme non-functional. HMG-CoA reductase is responsible for the rate-limiting step of HMG-CoA conversion to mevalonate in the cholesterol biosynthesis pathway [8]. Lovastatin also has pleiotropic application apart from its hypocholesterimic activity. Other applications include treatment of bone fracture, inflammation, diabetes, and chemoprevention. However, the oral administration of this drug has certain limitations, viz., poor bioavailability, nonhepatic shunting, side effects due to higher dosage, more excretion in bile. Inactive lactone (prodrug) and its active $\beta$ hydroxy acid metabolite have short half-lives (1-2 h) [6]. 
Cholesterol biosynthesis in non-hepatic cells is important in normal cellular activities. Non-hepatic shunting of this drug leads to severe problems. Another important parameter is lipophilicity which determines the nature of the diffusion system. The lactone form of this drug is more lipophilic than its hydroxy acid form. This helps the lactone form to display efficient hepatic shunting through a simple passive diffusion mechanism. A formulation with a high degree of absorption and extended delivery potential would be extremely desirable for lovastatin [6]. Hence, this compound is encapsulated or coated with a suitable carrier to enhance its bioavailability and other properties, viz., dissolution rate, half-life period, drug loading capacity, and maximum attainable plasma concentration. Drug design will help to find more new pleiotropic applications with fewer side effects through the elimination of various potential barriers. This paper discusses emerging formulation designs for the delivery of poorly water-soluble lovastatin, their characterization, and applications.

\section{LIPID-BASED CARRIERS FOR LOVASTATIN}

Drugs are introduced into the human system with suitable carriers to increase their efficiency in different physiological conditions of the host system. Carriers like lipid-based carriers, polymeric supports, quantum dots, and microparticles are available for targeted drug delivery [3]. Lipid-based carriers are chosen, especially in lovastatin nanoparticles synthesis, due to some of the drawbacks of all other supporting materials. Lovastatin solid lipid nanoparticles (SLNs) are one such example for lipid-based carriers made from solid lipids. The solid lipids remain solid at body temperature and are stabilized by suitable surfactant(s). These lipidic materials can be highly purified triglycerides, complex glyceride mixtures or even waxes $[6,9,10]$. These SLNs have their own merits, such as lower cytotoxicity, excellent physical stability, protection of incorporated labile drugs from degradation, controlled drug release (fast or sustained depending on the incorporation method), good tolerance, site-specific targeting, physico-chemical properties, and best production scalability [8]. The inborn potential demerits of these SLNs are insufficient drug loading capacity, drug expulsion after polymorphic transition during storage, and relatively high water content of the dispersions $(70-99.9 \%)[2,6,9,10]$. The perfection in the resultant nanoparticles is mainly dependent on the lipid matrix composition. A perfect crystal with few imperfections might be possible only if the lipid matrix consists of similar molecules. A highly-ordered crystal lattice cannot accommodate high amounts of drug since the incorporated drug can be located between fatty acid chains, between the lipid layers, and also in crystal imperfections. Therefore, the use of more complex lipids such as mono, di, and triglycerides with different chain lengths are more suitable for higher drug loading. The transition to highly-ordered lipid particles could also be the reason for drug expulsion from the prepared nanoparticles. Usually, the synthesized lipids were partially crystallized in higher energy modifications with more imperfections in the crystal lattice. Moreover, the preservation of the $\alpha$-modification during storage and transformation after administration (e.g., by temperature changes) could lead to triggered and controlled release characteristics. This modification depends on change in temperature. More reports are available on these aspects for topical formulations [2, 6, 9, 10]. Lipid emulsions (LEs), as reported, have been prepared using liquid lipids and coated with liquid emulsifiers. Besides the merits, it has more demerits like physical instability caused by both the incorporated drug and the storage environment. Lipid-drug conjugates and liposomes are also found to have potential medicinal applications $[3,6,11]$.

Another wide and advanced generation lipidic carrier is the nanostructured lipid carrier (NLCs) which possesses a solid lipid matrix and a portion of suitable liquid lipid in the core side along with the lactone form of this drug. The efficiency of the NLCs was improved by a lipophilic emulsion coating. The NLC system has been shown to have more oral administration bioavailability, physical stability, improved properties for drug loading, modulation of drug delivery profile, and stable drug incorporation during storage. Due to its lipophilic nature, it is well-suited for lipophilic drug administration. Some of the lipophilic and hydrophobic emulsifiers used for the preparations of NLCs are myverol 18-04K, soybean phosphatidylcholine, and Pluronic F68 [6, 10]. These emulsifiers play a vital role in targeted drug delivery, stabilization during storage, and optimum release of this drug [6, 7].

NLCs are further classified based on their solid and liquid lipid content, spatial arrangement of the drug, dispersing (aqueous or oil) medium, and composition. One such classification based on the composition of lipids are low oil, high oil, and amorphous oil NLCs [7, 11]. More space for the accommodation of drug molecules (high drug load) could be achieved by mixing solid lipids with small amounts of liquid lipids (oils). For example, spatially different lipids like glycerides composed of different fatty acids can be mixed together leading to larger distances between the fatty acid chains of the glycerides and general imperfections in the crystal. This model is known as "imperfect type NLCs" [7]. Many drugs show a higher solubility in oils than in solid lipids. Hence, this drug can be dissolved in oil and still be protected from degradation by the surrounding solid lipids. The amount of oil should not exceed the amount of solid lipid to get a perfect nanostructure. Since a high solubility of oil molecules in the solid lipid leads to phase separation, and there is a possibility of the formation of oily nanocompartments within the solid lipid matrix [7, 9]. This "multiple-type NLC'" can be considered as an analog to water/oil/water emulsion as it has oil-in-solid lipid-in-water dispersion. Usually, drug expulsion was noticed either during crystallization or transformation of the solid lipid. This can be prevented by the formation of an "amorphous type NLC', [9]. Hydroxyoctacosanylhydroxystearate and isopropylmyristate are the special lipids used for crystallization instead of natural cooling. It has been used as a carrier for various drug targeting systems due to its potential merits [6, $7,11]$.

\section{PREPARATION OF LIPID-BASED CARRIERS}

A suitable lipid-based carrier for this drug was prepared by choosing suitable lipidic materials (liquid and solid), processing conditions, and processes (Fig. 1). Usually, lipidic carriers are chosen for lipophilic drugs mainly due to their poor solubility characteristics. Solid lipids are used to give a solid support to the incorporated drug. Solid lipids are 


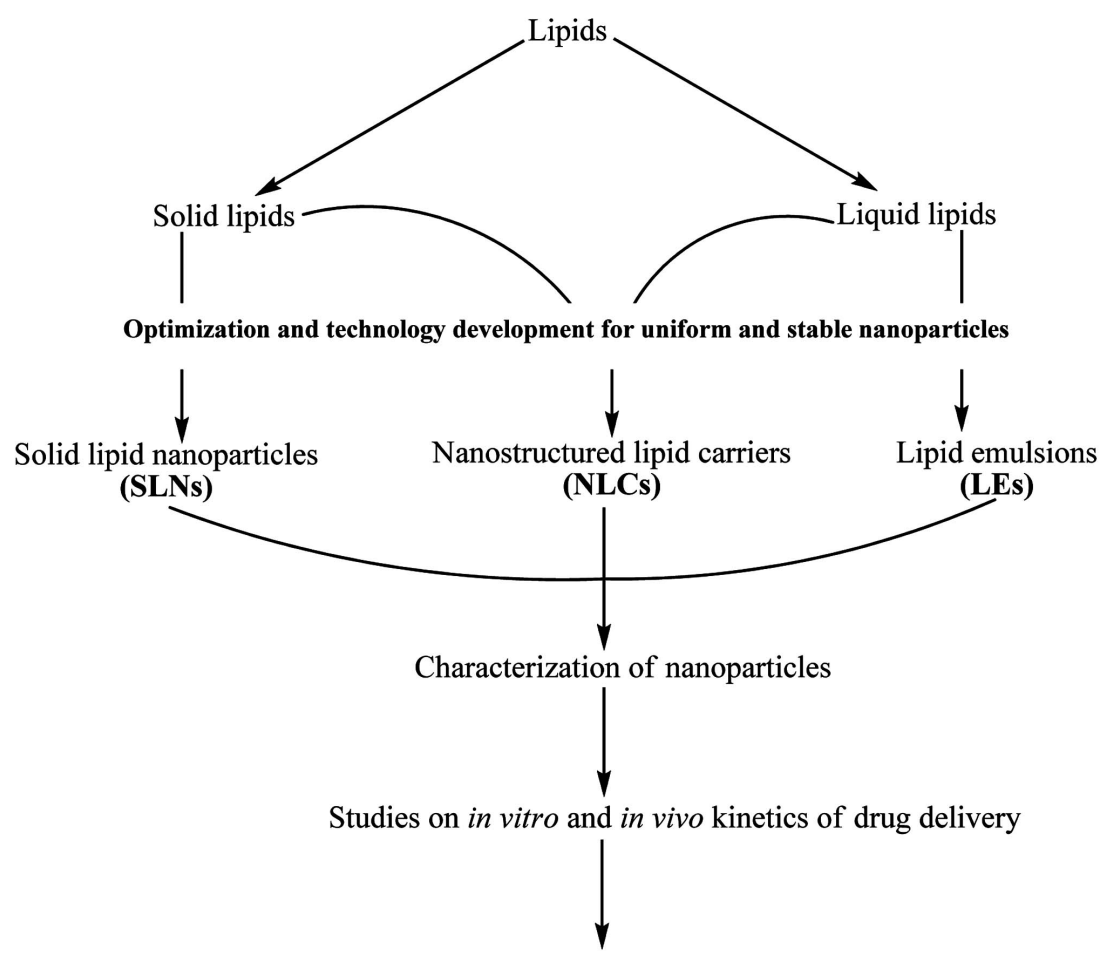

Clinical implementation

Fig. (1). Schematic illustration of the overall process involved in lovastatin nanoparticles preparation and characterization

melted to prepare the solid coating over the drug particles or to entrap the nanoparticles based on the immobilization techniques employed for enzymes and organisms. Initially, the lactone form of this drug powder was mixed with lipidic materials, viz., triglyceride and phosphatidylcholine and then dissolved in a suitable mixture of solvent. The selection and type of lipidic materials is important and is dependent on the type of application. Usually the SLNs were prepared by mixing this drug with a suitable solid lipid. Similarly, the lipid emulsions were prepared by mixing relatively low lipidic material with an aqueous emulsifying agent. The temperature of both the oil and aqueous phases should be the same to enhance mixing. Since these emulsifying agents determine the modulation in drug solubility and efficiency, the choice and difference in composition of the emulsifying agents are crucial factors which need to be considered for optimal design and delivery of nanoparticles. After mixing the hot aqueous phase and the oil phase together, the resulting solution was homogenized to a proper hot oil-in-water emulsion. This emulsion was then subjected to ultrasonication at both low and high frequencies to reduce the size of the nanoparticles and for proper homogenization. Finally, the desired nanoparticles were obtained by allowing the hot nanoemulsion to cool at room temperature. By this procedure, various lipidic carriers have been prepared for many drugs and especially for this lipophilic drug $[2,6,10]$.

Various methodologies such as high-pressure homogenization, preparation via micro-emulsions, solvent emulsification-evaporation or -diffusion, water-oil-water double emulsion, ultrasonication or high-speed stirring, precipitation with a compressed fluid anti-solvent system, rapid expansion from a liquefied-gas solution, spray freezing into liquid, and evaporative precipitation into aqueous solution are employed to produce specialized nanoparticles $[1,6,7,11]$. Various combinations of solid, liquid lipids, and suitable emulsifiers have been employed to get optimum and more effective nanoparticles. For example, new generation NLCs were prepared by mixing both the solid and liquid lipids in different compositions to get an optimal delivery profile. There are some successful technologies employed in the preparation of specialized nanoparticles for delivering poorly soluble drugs to its target sites. Generally, these different technologies and their development are based on specific drug requirements such as barriers to its target, sustained release characteristics, sensitivity, physico-chemical properties, and expected modulation profiles. Moreover, the formation of cavities, nature of drug and its stability, position, drug loading capacity, melting point of the active ingredients, dissolution, precipitation properties, and final product characteristics are also important in the technology development. These technologies have to be robust and should be applicable to various drugs in different environments. Examples for some selective technologies available in the preparation of drug carriers are dissocubes, nanocrystal technology, nanomorph technology, nanoedge technology, nanopure technology, crititech technology, nanocochleate technology, and controlled-flow cavitation technology $[1,6,7,11]$. These technologies can be adapted for lovastatin nanoparticle synthesis with necessary modifications.

\section{CHARACTERIZATION OF LIPID-BASED CARRI- ERS}

Many techniques are available for the characterization of lovastatin nanoparticles. The drug sample was subjected to hot homogenization followed by ultrasonication with and without the lipid material to get the desired size. It is important to have nanoparticles of the desired size, bioavailability, and physico-chemical stability. Size measurement is a cru- 
cial factor by which design processes are optimized. The average size and zeta potential were measured by photon correlation spectroscopy at a particular temperature $\left(25^{\circ} \mathrm{C}\right)$ and scattering angle $\left(90^{\circ}\right)$. The zeta potential measurement of lovastatin is based on its electrophoretic mobility in the aqueous medium $[6,10]$. Suresh et al. have studied the average size and zeta potential of lovastatin nanoparticles at $25^{\circ} \mathrm{C}$ at an electric field strength of $23.2 \mathrm{~V} / \mathrm{cm}$ at different $\mathrm{pH}$ values ( $\mathrm{pH} 6.9$ to 7.2). They determined the average size and zeta potential of SLNs (99.5\% encapsulation efficiency) as $65.6 \mathrm{~nm}$ and $-15.8 \mathrm{mV}$, respectively with tripalmitin [10]. Chen et al. studied the effect of emulsifying agents on different combinations of NLCs. They reported an average size and zeta potential of $183.2 \mathrm{~nm}$ and $-2.7 \mathrm{mV}$, when soybean phosphatidylcholine and myverol emulsifiers were used with precirol:squalene in the ratio of $2: 8$ and $0: 10$, respectively. Moreover, the nanoparticles with consistent negative charge could not be controlled with the lipidic material and emulsifiers [6]. Encapsulation efficiency is another crucial parameter that must be measured to find the effectiveness of lipid materials and process parameters involved in various operations, viz., homogenization, ultrasonication, and mixing. The non-encapsulated lovastatin was removed by size exclusion chromatography from the prepared stock of nanoparticles and the encapsulated drug concentration was measured by HPLC $(238 \mathrm{~nm})$ by dissolving the coating over this drug. Maximum encapsulation efficiencies of $73 \%$ and $65 \%$ were obtained with myverol and soybean phosphatidylcholine, respectively [6]. The degree of crystallinity was measured by differential scanning calorimetry (DSC) and powder X-ray diffractometry (PXRD) which also helped investigate the status of the lipids and the drug in the prepared and lyophilized nanoparticles preparation [10].

The molecular environment (polarity) could be ascertained by fluorometric spectrophotometry based on the solvatochromism of Nile red, a lipophilic fluorescent marker used as the model solute. Partitioning of the drug in both the lipid and aqueous phases was measured by HPLC to know the amount of drug in both the phases [6]. In vitro release characteristics of this drug from lipid nanoparticles were measured using a Franz diffusion cell system and the quantum of drug released from the carriers due to the environmental conditions was measured by HPLC. An in vivo study has been reported in animals to determine the pharmacokinetic characteristics of this drug by measuring its concentration in the blood serum using HPLC [6]. Recently, animal skin has been used in the Franz diffusion cell to study the permeability characteristics and other important pharmacological parameters. Apart from these analyses, the stability of the prepared nanoparticles was studied by measuring the particle size (photon correlation spectroscopy, laser diffraction), charge, and thermal analysis (DSC) as a function of time $[6,10]$. These analyses gave the result of any decay or expulsion or physical deformation in the prepared nanoparticles during preparation and storage. The stability of the nanoparticles determines the overall success of the technology. Other analyses, viz., biological, radiographic techniques and immunological safety analysis would be necessary to estimate the target-determining capacity, transport characteristics, and toxicity of the prepared nanoparticles $[3,12]$.

\section{CONCLUSIONS}

Nanoparticle-based technologies enhance certain properties of this poorly water-soluble drug, lovastatin, viz., drugloading efficiency, stability, efficient first-pass uptake into hepatic cells, less toxicity, faster excretion, maximum plasma concentration, and bioavailability. Carriers must be chosen carefully and must be designed for different environments and applications to enhance all possible pleiotropic effects. In the context of pleiotropic application, this drug delivery system has shown better results compared to conventional treatment in the case of bone fracture. Some of the characteristics of this lipophilic drug, viz., target specificity, diffusion, sustained release, and intactness can be improved by careful selection of the lipidic material. LEs, SLNs, and NLCs are some of the diversified carriers used in drug delivery system. Among these, NLCs have lots of advantages over the other carriers, such as high drug loading capacity, improved release characteristics, and possibility of multiple drug incorporation. The conventional concept of "one drug and one disease" model can be overcome by incorporating multiple drugs including vitamins and antioxidants in a layer by layer fashion. Still, studies on this lovastatin-based nano-aided system for other applications are not available. The impact of residual solvent is an important problem that needs to be addressed by a careful selection of the dispersion medium or by the use of supercritical fluids in a green way so as to avoid harmful effects to the host system. Potential problems of the drug delivery system have been addressed by engineering the lipid materials, emulsifiers, and dispersion medium in a green way. This robust system is well-suited for drugs having high sensitivity, site specificity, and is highly toxic to sites other than the target site. Thus, the nano-aided drug delivery system is an appropriate option for poorly soluble lipophilic drugs.

\section{REFERENCES}

[1] Fahr A, Liu X. Drug delivery strategies for poorly water soluble drugs. Expert Opin Drug Deliv 2007; 4(4): 403-16.

[2] Blasi P, Giovagnoli S, Schoubben A, Ricci M, Rossi C. Solid lipid nanoparticles for targeted brain drug delivery. Adv Drug Deliv Rev 2007; 59(6): 454-77.

[3] Puri A, Loomis K, Smith B, et al. Lipid-based nanoparticles as pharmaceutical drug carriers: from concepts to clinic. Crit Rev Ther Drug Carrier Syst 2009; 26(6): 523-80.

[4] Pardeike J, Hommoss A, Müller RH. Lipid nanoparticles (SLN, NLC) in cosmetic and pharmaceutical dermal products. Int J Pharm 2009; 366: 170-84

[5] Kaur IP, Bhandari R, Bhandari S, Kakkar V. Potential of solid lipid nanoparticles in brain targeting. J Control Release 2008; 127(2): 97-109.

[6] Chen CC, Tsai TH, Huang ZR, Fang JF. Effects of lipophilic emulsifiers on the oral administration of lovastatin from nanostructured lipid carriers: physicochemical characterization and pharmacokinetics. Eur J Pharm Biopharm 2010; 74: 74-82.

[7] Müller RH, Petersen RD, Hommoss A, Pardeike J. Nanostructured lipid carriers (NLC) in cosmetic dermal products. Adv Drug Deliv Rev 2007; 59(6): 522-30.

[8] Seenivasan A, Subhagar S, Aravindan R, Viruthagiri T. Microbial production and biomedical applications of lovastatin. Indian J Pharm Sci 2008; 70:701-9.

[9] Wissing SA, Kayser OA, Müller RH. Solid lipid nanoparticles for parenteral drug delivery. Adv Drug Deliv Rev 2004; 56(9): 1257 72.

[10] Suresh G, Manjunath K, Venkateswarlu V, Satyanarayana V. Preparation, characterization, and in vitro and in vivo evaluation of lovastatin solid lipid nanoparticles. AAPS PharmSciTech 2007; 8(1): E162-70. 
[11] Radtke M, Souto EB, Müller RH. Nanostructured lipid carriers: a novel generation of solid lipid drug carriers. PharmTech Eur 2005; 17(4): 45-50.
[12] Garrett IR, Gutierrez GE, Rossini G, et al. Locally delivered lovastatin nanoparticles enhance fracture healing in rats. J Orthop Res 2007; 25(10): 1351-7.

Received: May 20, 2011

Revised: June 10, 2011

Accepted: June 13, 2011

(C) Seenivasan et al.; Licensee Bentham Open.

This is an open access article licensed under the terms of the Creative Commons Attribution Non-Commercial License (http://creativecommons.org/licenses/by-nc/3.0/) which permits unrestricted, non-commercial use, distribution and reproduction in any medium, provided the work is properly cited. 